\title{
PIK3CA-CDKN2A clonal evolution in metastatic breast cancer and multiple points cell-free DNA analysis
}

\author{
Maria Palmieri ${ }^{1}$, Margherita Baldassarri ${ }^{2}$, Francesca Fava' ${ }^{1}$, Alessandra Fabbiani', Giuseppe Maria Campenni ${ }^{3}$, \\ Maria Antonietta Mencarelli ${ }^{2}$, Rossella Tita ${ }^{2}$, Stefania Marsili ${ }^{4}$, Alessandra Renieri ${ }^{1,2^{*}}$ and Elisa Frullanti ${ }^{1}$
}

\begin{abstract}
Background: Daily experience tells us that breast cancer can be controlled using standard protocols up to the advent of a relapse. Now new frontiers in precision medicine like liquid biopsy of cell free DNA (cfDNA) give us the possibility to understand cancer evolution and pick up the key mutation on specific cancer driver gene. However, tight schedule of standardized protocol may impair the use of personalized experimental drugs in a timely therapeutic window.

Main body: Here, using a combination of deep next generation sequencing and cfDNA liquid biopsy, we demonstrated that it is possible to monitor cancer relapse over time. We showed for the first time the exact correspondence from the increasing clonal expansion and clinical worsening of metastatic breast cancer.

Conclusion: Thanks to liquid biopsy may be possible to introduce new experimental drugs in the correct therapeutic window which would lead in the near future to an effective treatment which otherwise remains challenging.
\end{abstract}

Keywords: PIK3CA-CDKN2A, cfDNA, Liquid biopsy, Deep-next generation sequencing, Targeted-therapy

\section{Background}

Melchardt et al. 2018 demonstrated that clones in distant relapse of head and neck cancer are different in respect to those identified at the beginning in tumor biopsy [1]. Classically, haematological malignancies have taught us that, within the dynamic clonal evolution, a subclonal expansion of a pre-existing mutated clone leads often to relapse [2, 3]. Expanding clones may be selected by treatment acquiring drug resistance and patients who relapse after an effective therapy usually have a poor prognosis $[4,5]$. While at the beginning of tumor expansion there is a consistent, although variable, mutational burden from ten to hundred clones, at relapse the leading clone is usually only one [5]. Liquid biopsy of cell free DNA (cfDNA) has now the potential to follow the temporal evolution and to inform us about the driver mutation of

\footnotetext{
*Correspondence: alessandra.renieri@unisi.it

1 Medical Genetics Unit, Policlinico "Santa Maria alle Scotte", University of Siena, Viale Bracci, 2, 53100 Siena, Italy

Full list of author information is available at the end of the article
}

the expanded clone. Among solid tumors, breast cancer is one of the most facing a high risk of recurrence after curative surgery and therapeutic treatment. PIK3CA mutations are found in $27 \%$ of cases of disease progression in breast cancer [6]. Free survival was demonstrated to be inversely correlated with PIK3CA mutations at liquid biopsy [7] but longitudinal analysis of clonal evolution and disease progression was missed.

\section{Main text}

Using a combination of deep next generation sequencing and cfDNA liquid biopsy (Oncomine pan-cancer cell free assay and tissues/blood custom panels on ion proton platform, life technologies), we showed for the first time the exact correspondence between the increasing clonal expansion and the clinical worsening of metastatic breast cancer in a 44-year-old female with disease recurrence after 4 years and half of disease control. At 38 years she presented a poorly differentiated ductal carcinoma of the right breast, pT1cN1mi(1/3) G3, estrogen receptor (ER) 
positive $90 \%$, progesterone receptor (PgR) positive 40\%, HER2 negative, KI67 40\%. She underwent quadrantectomy and subsequent chemotherapy with Epirubicin Ciclofosfamide (EC) for four cycles and Decapeptyl every 28 days. After 7 months, she added tamoxifen for 1 year, then replaced by Exemestrane for 2 years. Radiotherapy was also performed. Family history was positive for breast cancer in the paternal side although exome analysis failed to reveal germline mutation in known cancer driver genes.

After 4 years and half from the diagnosis, liver and bone metastasis were detected. Biopsy of the VIII hepatic segment showed metastasis of breast carcinoma, ER negative, PgR negative, HER2 negative, Ki67 17\%. Subsequently, therapy with Paclitaxel was undertaken weekly. Three months later from the previous evidence of metastasis, numerical and dimensional increase in liver lesions was demonstrated. Monotherapy with cisplatin was started and then in association with capecitabine and epirubicin (ECX scheme) with dose of $80 \%$ for seven consecutive cycles. Afterwards, she had treatment with
Capecitabine and metronomic cyclophosphamide for 3 months, then three cycles of eribuline were performed. Therapy with carboplatin and gemcitabine was subsequently administered. One month later, since there was an increase of liver enzymes, monotherapy with carboplatin was undertaken. No other treatment options were available given the disease progression.

After about 1 year from the evidence of bones and liver metastasis, we performed a first cfDNA analysis, which revealed pathogenic mutations in PIK3CA gene [c.1633G >A; p.(Glu545Lys)] and CDKN2A [c.1904T>G; p.(Leu635Arg)]. Five months later, a second cfDNA analysis highlighted an exponential increase of clones with the same pathogenic mutations (Fig. 1). In comparison with the mutational burden identified at primary tumor biopsy, the expanding clone has a simplified architecture (Fig. 1).

We demonstrated here that multiple points cfDNA analysis give now the possibility to understand the overall cancer dynamics and pick up the key mutation leading to cancer recurrence, separating them from not expanding

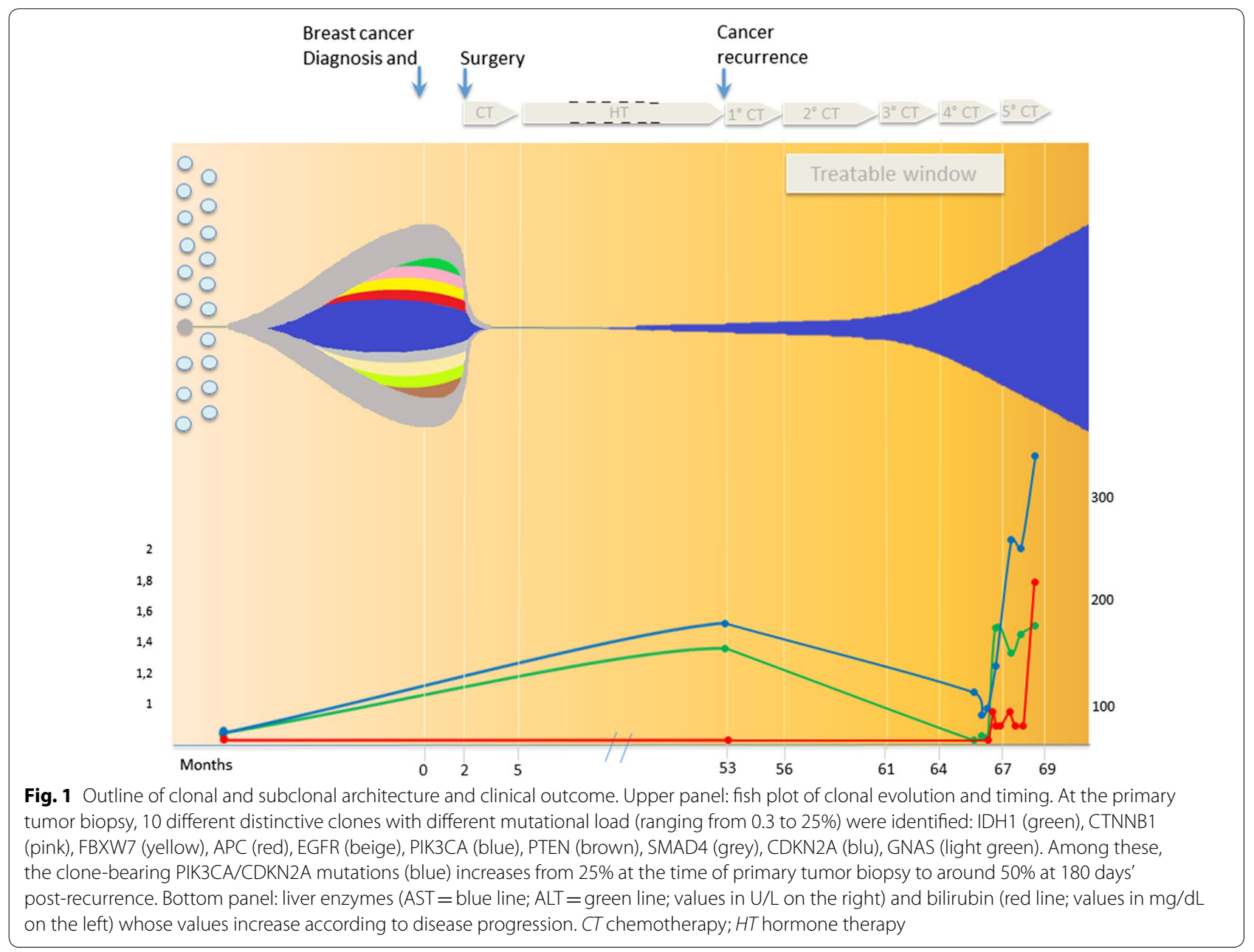


occasional clones. Large comprehensive analysis of haematological malignancies indicates a complex temporal dynamic landing although in few (from one to six) driver mutations at late relapse [8]. Likewise, in breast cancer one (or few) is the driver mutation leading to cancer recurrence and it is of pivotal importance to target this driver mutation(s) on time. However, stick too tightly to standardised protocol may impair the use of personalised experimental drugs in a timely therapeutic window.

Data reported here indicate that there is a tight therapeutic window useful for counteract final clonal expansion and that the minimally invasive cfDNA analysis allows a close and dynamic monitoring of clonal evolution. This is also supported by the mathematical model developed by Khan et al. [9]. In our case, an innovative and more effective therapy could be CDK $4 / 6$ inhibitors in combination with PI3 K-specific inhibitor initiated at the beginning of the clonal expansion [10]. Introducing of experimental drug in the correct therapeutic window would lead in the near future to effective treatment which otherwise remain challenging.

\section{Conclusions}

In conclusion, we demonstrated that multiple points cfDNA analysis reflects clonal evolution and allows track the evolving molecular landscapes of growing cancer cells by capturing broader molecular alterations that could hinder targeted treatments efficacy. The shorter turnaround time of cfDNA analysis and its high sensitivity and specificity are key factors to provide novel opportunities for adaptive personalised therapies, optimizing healthcare resources and enabling higher treatment efficacy and lower side-effects.

\footnotetext{
Abbreviations

CDKN2A: cyclin dependent kinase inhibitor 2A; CDK: cyclin dependent kinase; cfDNA: cell-free DNA; EC: epirubicin ciclofosfamide; ECX: epirubicin cisplatin and capecitabine; ER: estrogen receptors; HER2: human epidermal growth factor receptor 2; PgR: progesterone receptor; PIK3CA: phosphoinositide-3-kinase, catalytic, alpha polypeptide.
}

\begin{abstract}
Acknowledgements
The cell lines and DNA bank of Rett Syndrome, X-linked mental retardation and other genetic diseases, member of the Telethon Network of Genetic Biobanks (project no. GTB12001), funded by Telethon Italy, and the EuroBioBank network, provided us with specimens. Authors also thank the ERN-EURACAN (European network for Rare adult solid Cancer). We thank SienaGenTest srl, a Spin-off of the University of Siena (www.sienagentest.dbm. unisi.it) for bioinformatic analysis of genetic data.
\end{abstract}

\section{Authors' contributions}

MP performed experiments on cfDNA and analysed the data and wrote the paper, FF, AF, took care of the clinical part of the study and wrote the paper, RT performed experiments on blood, tumoral tissue and metastasis, AR designed the research strategy, analysed the data and wrote the paper, EF analysed the data and wrote the paper, MB and MAM performed genetic counselling and provided patient samples, SM and GMC followed the patient as oncologist. All authors read and approved the final manuscript.

\section{Funding}

Authors would thank for support Regione Toscana - Istituto Toscano Tumori (ITT) (Project "Identification of genetic bases of individual predisposition to lung cancer in non-smokers") and from ASSO (Associazione per lo Sviluppo della Scienza Oncologica) onlus.

\section{Availability of data and materials}

The datasets used and/or analyzed during the current study are available from the corresponding author on reasonable request.

\section{Ethics approval and consent to participate}

This study was consistent with Institutional guidelines and approved by the ethical committees of Azienda Ospedaliera Universitaria Senese, Siena, Italy. Informed consent was obtained from the patient.

\section{Consent for publication}

Consent was obtained from the patient.

\section{Competing interests}

The authors declare that they have no competing interests.

\section{Author details}

${ }^{1}$ Medical Genetics Unit, Policlinico "Santa Maria alle Scotte", University of Siena, Viale Bracci, 2, 53100 Siena, Italy. ${ }^{2}$ Genetica Medica, Azienda Ospedaliera Universitaria Senese, Siena, Italy. ${ }^{3}$ European Institute of Oncology, Milan, Italy. ${ }^{4}$ Oncology, Azienda Ospedaliera Universitaria Senese, Siena, Italy.

Received: 27 May 2019 Accepted: 12 October 2019

Published online: 28 October 2019

\section{References}

1. Melchardt T, Magnes T, Hufnagl C, Thorner AR, Ducar M, Neureiter D, et al. Clonal evolution and heterogeneity in metastatic head and neck canceran analysis of the Austrian Study Group of Medical Tumour Therapy study group. Eur J Cancer. 2018. https://doi.org/10.1016/j.ejca.2018.01.064.

2. Fouillet L, Daguenet E, Schein F, Tavernier E, Flandrin-Gresta P, Cornillon J. Clonal evolution of myelofibrosis treated with hematopoietic transplantation, using RUXOLITINIB for chronic GVHD: a case report. Curr Res Transl Med. 2018. https://doi.org/10.1016/j.retram.2018.07.001.

3. Pinto AM, Papa FT, Frullanti E, Meloni I, Tita R, Caselli R, et al. Low-level TP53 mutational load antecedes clonal expansion in chronic lymphocytic leukaemia. Br J Haematol. 2019. https://doi.org/10.1111/bjh.15147.

4. Ghatalia P, Smith CH, Winer A, Gou J, Kiedrowski LA, Slifker M, et al. Clinical utilization pattern of liquid biopsies (LB) to detect actionable driver mutations, guide treatment decisions and monitor disease burden during treatment of 33 metastatic colorectal cancer (mCRC) patients (pts) at a Fox Chase Cancer Center Gl Oncology Subspecialty Clinic. Front Oncol. 2019. https://doi.org/10.3389/fonc.2018.00652.

5. Jacoby MA, Duncavage EJ, Chang GS, Miller CA, Shao J, Elliott K, et al. Subclones dominate at MDS progression following allogeneic hematopoietic cell transplant. JCI Insight. 2018. https://doi.org/10.1172/jci.insig ht.98962.

6. Lee JH, Jeong H, Choi JW, Oh HE, Kim YS. Liquid biopsy prediction of axillary lymph node metastasis, cancer recurrence, and patient survival in breast cancer. Medicine. 2018. https://doi.org/10.1097/MD.0000000000 012862.

7. Nakauchi C, Kagara N, Shimazu K, Shimomura A, Naoi Y, Shimoda M, et al. Detection of TP53/PIK3CA mutations in cell-free plasma DNA from metastatic breast cancer patients using next generation sequencing. Clin Breast Cancer. 2016. https://doi.org/10.1016/j.clbc.2016.05.004.

8. Spinella JF, Richer C, Cassart P, Ouimet M, Healy J, Sinnett D. Mutational dynamics of early and late relapsed childhood ALL: rapid clonal expansion and long-term dormancy. Blood Advances. 2018. https://doi. org/10.1182/bloodadvances.2017011510.

9. Khan KH, Cunningham D, Werner B, Vlachogiannis G, Spiteri I, Heide $T$, et al. Longitudinal liquid biopsy and mathematical modeling of clonal evolution forecast time to treatment failure in the PROSPECT-C 
phase II colorectal cancer clinical trial. Cancer Discov. 2018. https://doi. org/10.1158/2159-8290.CD-17-0891.

10. Gul A, Leyland-Jones B, Dey N, De P. A combination of the PI3 K pathway inhibitor plus cell cycle pathway inhibitor to combat endocrine resistance in hormone receptor-positive breast cancer: a genomic algorithmbased treatment approach. Am J Cancer Res. 2018;8:2359-76.

\section{Publisher's Note}

Springer Nature remains neutral with regard to jurisdictional claims in published maps and institutional affiliations.
Ready to submit your research? Choose BMC and benefit from:

- fast, convenient online submission

- thorough peer review by experienced researchers in your field

- rapid publication on acceptance

- support for research data, including large and complex data types

- gold Open Access which fosters wider collaboration and increased citations

- maximum visibility for your research: over $100 \mathrm{M}$ website views per year

At BMC, research is always in progress.

Learn more biomedcentral.com/submissions 